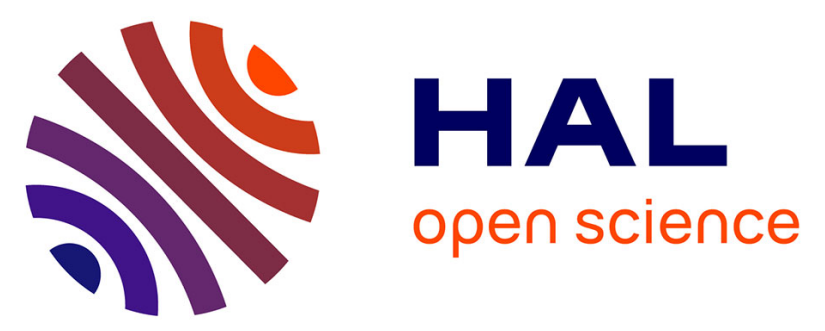

\title{
Autoinflammation secondaire à des défauts d'ubiquitination dans la voie NFKB : haploinsuffisance de A20 (HA20) et déficit en Otuline (Otulinopénie)
} Isabelle Koné-Paut, Sophie Georgin-Lavialle, Caroline Galeotti, Linda Rossi-Semerano, Véronique Hentgen, Léa Savey, David Saadoun, Guillaume Sarrabay, Isabelle Touitou

\section{To cite this version:}

Isabelle Koné-Paut, Sophie Georgin-Lavialle, Caroline Galeotti, Linda Rossi-Semerano, Véronique Hentgen, et al.. Autoinflammation secondaire à des défauts d'ubiquitination dans la voie NFKB : haploinsuffisance de A20 (HA20) et déficit en Otuline (Otulinopénie). Revue du Rhumatisme, 2019, 86, pp.358 - 366. 10.1016/j.rhum.2018.09.012 . hal-03486217

\section{HAL Id: hal-03486217 https://hal.science/hal-03486217}

Submitted on 20 Dec 2021

HAL is a multi-disciplinary open access archive for the deposit and dissemination of scientific research documents, whether they are published or not. The documents may come from teaching and research institutions in France or abroad, or from public or private research centers.
L'archive ouverte pluridisciplinaire HAL, est destinée au dépôt et à la diffusion de documents scientifiques de niveau recherche, publiés ou non, émanant des établissements d'enseignement et de recherche français ou étrangers, des laboratoires publics ou privés.

\section{(ㄷ)(1) $\$$}

Distributed under a Creative Commons Attribution - NonCommerciall 4.0 International 
Autoinflammation secondaire à des défauts d'ubiquitination dans la voie NFKB : Haploinsuffisance de A20 (HA20) et déficit en Otuline (Otulinopénie)

Isabelle Koné-Paut ${ }^{\text {a,b,c }}$, Sophie Georgin Lavialle ${ }^{\text {c,de,ff }}$, Caroline Galeotti ${ }^{\text {a,b,c }}$, Linda Rossi Semerano ${ }^{\mathrm{a}, \mathrm{b}, \mathrm{c}}$, Véronique Hentgen ${ }^{\mathrm{c}, \mathrm{g}}$, Léa Savey ${ }^{\mathrm{c}, \mathrm{d}, \mathrm{e,f}}$, David Saadoun ${ }^{\mathrm{c}, \mathrm{d}, \mathrm{h}, \mathrm{i}, \mathrm{j}}$, Guillaume Sarrabay ${ }^{\mathrm{c}, \mathrm{k}, \mathrm{l}}$, Isabelle Touitou ${ }^{\mathrm{c}, \mathrm{k}, \mathrm{l}}$.

${ }^{a}$ Service de rhumatologie pédiatrique, CHU de Bicêtre, Assistance publique-hôpitaux de Paris, 94270 Le Kremlin-Bicêtre, France

${ }^{\mathrm{b}}$ université de Paris SUD, 94270 Le Kremlin-Bicêtre, France

c Centre national de référence des maladies auto-inflammatoires et de l'amylose inflammatoire (CEREMAIA), 94270 Le Kremlin-Bicêtre, France

${ }^{\mathrm{d}}$ Sorbonne Universités, 75006 Paris, France

'Service de médecine interne, Département HospitaloUniversitaire InflammationImmunopathologie-Biotherapie (DHU i2B). hôpital Tenon, Assistance publique-hôpitaux de Paris (AP-HP), 4, rue de la Chine, 75020 Paris, France

f Laboratoire INSERM U_933. 75012 Paris, France

${ }^{g}$ Service de pédiatrie générale, Centre hospitalier de Versailles, 179, rue de Versailles, 78150 Le Chesnay, France

h Département de Médecine Interne et Immunologie Clinique, UMR 7211, Département HospitaloUniversitaire Inflammation-Immunopathologie-Biotherapie (DHU i2B), AP-HP, Groupe Hospitalier Pitié-Salpêtrière, 75005, Paris, France.

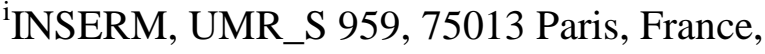

${ }^{\mathrm{j}} \mathrm{CNRS}$, FRE3632, 75005 Paris, France

${ }^{\mathrm{k}}$. Cellules souches, plasticité cellulaire, médecine régénératrice et immunothérapies, INSERM, Université de Montpellier, 34090 Montpellier, France

${ }^{\mathrm{l} D e ́ p a r t e m e n t ~ d e ~ g e ́ n e ́ t i q u e ~ m e ́ d i c a l e, ~ m a l a d i e s ~ r a r e s ~ e t ~ m e ́ d e c i n e ~ p e r s o n n a l i s e ́ e, ~ C H U ~}$ Montpellier, 34295 Montpellier, France

\section{Auteur correspondant :}

Pr Isabelle Koné-Paut : Service de rhumatologie pédiatrique, Centre de référence des maladies auto-inflammatoires et de l'amylose (CEREMAIA), CHU de Bicêtre, 78 rue du Général Leclerc, 94270 le Kremlin Bicêtre isabelle.kone-paut@aphp.fr 


\section{Résumé}

À côté des voies désormais classiques de l'autoinflammation que sont celles de l'interleukine1 (IL-1) et des interférons, le champ s'élargit avec la description de défauts de régulation négative de la voie NFKB. La voie NFKB, activée dès le contact d'un ligand sur le récepteur 1 du tumor necrosis factor (TNFR1), joue un rôle critique pour engager le processus inflammatoire car elle produit des cytokines majeures comme l'IL-1, l'interleukine 6 et le $\mathrm{TNF}$; sa régulation négative est essentielle à l'arrêt du processus inflammatoire et dépend du niveau d'ubiquitination des protéines associées au TNFR1 et d'autres composés intermédiaires. A20 et Otuline sont des protéines qui modifient l'ubiquitination et leur défaut peut entraîner une activation de la voie NFKB avec production excessive de cytokines proinflammatoires. L'haploinsuffisance d'A20 comme la maladie de Behçet inclut l'aphtose bipolaire et plus rarement l'uvéite, mais elle s'en distingue par sa transmission dominante, un début des symptômes plus précoce, et une atteinte digestive sévère au premier plan. Son hétérogénéité clinique est très importante, les patients atteints présentant au cours de leur vie de façon concomitante ou successive des manifestations autoinflammatoires, autoimmunes voire plus rarement un déficit immunitaire discret, essentiellement humoral. Le déficit en Otuline se caractérise par des manifestations systémiques inflammatoires très précoces associées à une panniculite avec lipodystrophie et une colite inflammatoire; son principal diagnostic différentiel étant le syndrome PRAAS : proteasome autoinflammatory associated syndrome. Le traitement de ces affections est difficile et non codifié à ce jour avec une corticosensibilité à haute dose, et une efficacité modeste des anti- TNF et des anti-IL1.

\section{Mots clés}

Autoinflammation, HA20, TNFAIP3, déficit en Otuline (ORAS), Otulinopenie, NFKB, Behçet, ubiquitination. 


\section{Introduction}

Les manifestations cliniques des maladies autoinflammatoires: poussées systémiques inflammatoires survenant apparemment de façon inopinée, sont dues à un défaut de régulation de l'immunité innée conduisant à une production excessive de cytokines proinflammatoires telles que l'interleukine-1 (IL-1), l'interleukine 18 (IL-18), l'interleukine 6 (IL-6), le TNF alpha $(\mathrm{TNF} \alpha)$ et les interférons de type I (IFN) [1-3]. Les maladies monogéniques associées à IL-1 ont été le premier groupe décrit et pour un bon nombre d'entre elles l'utilisation des biothérapies anti IL-1 s'est avérée une révolution pour la qualité et probablement aussi l'espérance de vie des patients aux phénotypes les plus sévères [2]. Au fil du temps et grâce à d'intenses collaborations cliniques et génétiques et du fait du développement des nouvelles techniques de séquençage à haut débit, de nouvelles voies d'autoinflammation sont régulièrement découvertes dont certaines ont des frontières floues avec dépassent les limites avec l'autoimmunité et le déficit immunitaire [1]. Cette revue éclaire sur les défauts de l'ubiquitination, un processus de modification post traductionnelle des protéines, qui modifie leur destin dans la cellule en leur rajoutant (ubiquitination) ou en leur retirant (déubiquitination) des résidus d'ubiquitine [4,5]. Ce mécanisme est particulièrement important dans le fonctionnement de la voie NFKB (TNF-nuclear factor-kB), une voie majeure de transcription de cytokines proinflammatoires. Des défauts de certaines protéines ayant des propriétés d'ubiquitination et/ou de déubiquitination (déubiquitinase : DUB) comme A20 et l'otuline, sont responsables respectivement de deux nouvelles entités autoinflammatoires, le syndrome HA20 (haploinsuffisance de TNFAIP3/A20) initialement décrit avec un phénotype assez proche de la maladie de Behçet (MB), mais qui avec le temps semble offrir un spectre clinique plus large alliant autoinflammation et autoimmunité ; et l'otulinopénie qui en plus de comporter une diarrhée chronique présente quelques similarités avec le syndrome PRAAS : proteasome autoinflammatory associated syndrome[4,6-13].

L'otuline est une déubiquitinase, qui hydrolyse les chaînes d'ubiquitine linéaires fixées par le complexe LUBAC (Linear Ubiquitin Chain Assembly Complex) [4,5,7,9]. Le complexe LUBAC est impliqué dans l'ubiquitination de deux éléments clés de la voie NFKB (RIPK1 et NEMO) et dont les mutations conduisent également à des syndromes autoinflammatoires avec déficit immunitaire [14,15]. Les deux déficits des DUBs: A20 et Otuline, sont donc 
responsables d'un défaut de régulation négative de la voie NFKB avec production excessive de cytokines proinflammatoires [4].

\section{Physiopathologie (Figure 1)}

\subsection{Ubiquitination}

L'ubiquitination est une modification post traductionnelle des protéines qui joue un rôle régulateur dans les processus de transduction des signaux d'activation en réponse à une stimulation via les récepteurs de l'immunité innée[4,5]. Sous stimulation, les chaînes d'ubiquitine sont assemblées puis attachées à la protéine dont elles doivent moduler la fonction et la destinée ; les chaînes d'ubiquitine existent sous différentes formes, en fonction d'où et comment elles se fixent; l'ubiquitination commence par l'attachement d'un simple motif d'ubiquitine à une protéine cible suivant un processus enzymatique en 3 étapes qui comportent : une enzyme activatrice d'ubiquitine (E1), une enzyme conjuguant l'ubiquitine (E2), puis une enzyme liant l'ubiquitine (E3 ligase)[4,5]. Les chaînes d'ubiquitine sont générées par l'attachement de monomères d'ubiquitine attachés sur des résidus qui sont soit des lysines (Lys-Ub; K48, K63) soit des méthionines (Met-Ub, Met1); les chaînes d'ubiquitine une fois liées peuvent subir des modifications ultérieures et/ou être détectées par des récepteurs ou des détecteurs qui vont fixer la destinée de leurs cibles [4]. Par exemple les enzymes marquées via des lysines en position 48 (K48) vont subir une dégradation de type UPS (ubiquitin-proteasome system) dans le protéasome; les déubiquitinases enlèvent les chaînes d'ubiquitine en les hydrolysant sur les protéines modifiées. A20, OTULIN, CYLD, et CEZANNE, sont des DUBs qui régulent négativement la voie NFKB [4,5]. Les polymorphismes de ces DUBs ont été associés variablement à des maladies autoimmunes, des cancers et des maladies neurodégénaratives [5,16-19].

\subsection{Haploinsuffisance de la protéine A20}

La protéine A20 est un régulateur négatif de la voie de signalisation du NFKB; des polymorphismes du gène TNFAIP3 ont été trouvés associés à diverses maladies autoimmunes: lupus, polyarthrite rhumatoide, psoriasis, maladie de Crohn et diabète de type 1[17]. La protéine A20 a une dualité de fonction : par son domaine OTU elle joue un rôle de DUB, et 
par son domaine ZF (sept doigts de zinc) elle a une fonction d'ubiquitination (E3 ligase) ; sa fonction DUB consiste à enlever des chaînes d'ubiquitine liées à K63 sur des protéines de signal comme TRAF6 (TNFR-associated factor 6) et RIPK1 (Receptor-interacting serine/threonine-protein kinase 1) dans la voie $\operatorname{NFKB}[4,17,18]$. Dans sa fonction ubiquitination elle lie des chaînes d'ubiquitine liées à K48 sur RIPK1 pour la diriger vers le protéasome pour dégradation ; l'haploinsuffisance de A20 résulte en la perte d'une des copies du gène A20 ; la protéine correspondante ne peut plus être produite en quantité suffisante ce qui altère sa fonction[4,8].

\subsection{Otulinopénie}

Le complexe LUBAC est une ubiquitinase (E3 ligase) chargée de maintenir la stabilité de nombreuses protéines régulatrices de l'immunité innée comme le récepteur 1 du TNF (TNFR1), les récepteurs toll-like (TLRs), le récepteur de l'interleukine 1 (IL-1R,) le CD40, et des inflammasomes (inflammasome receptor signaling complexes : RSCs) [4,14,15]. Sous l'effet de signaux proinflammatoires, le complexe LUBAC est recruté pour ajouter des chaînes d'ubiquitination linéaire à ses substrats que sont IKK (NEMO), RIPK1, RIPK2, IRAKs, MyD88, et ASC (figure 1). L'ubiquitination linéaire par le complexe LUBAC est indispensable à l'assemblage des inflammasomes[5]. Le complexe LUBAC est formé de HOIP (HOIL-1 interacting protein; RNF31) et de deux protéines accessoires HOIL 1 (hemeoxidized IRP2 ubiquitin ligase $1 ; R B C K 1$ ) et SHARPIN (heme-oxidized IRP2 ubiquitin ligase 1; RBCK1); L'otuline (gumby; FAM105B) est une protéine qui régule négativement la fonction d'ubiquitination linéaire du complexe LUBAC en effectuant une hydrolyse linéaire des chaînes d'ubiquitine liées par une Met1 [4,7,9].

Ainsi, lorsque des mutations touchent les gènes TNFAIP3 et OTULIN, codant respectivement les protéines A20 et otuline, le système d'ubiquitination/désubiquitination des protéines est perturbé et la voie NFKB peut être anormalement activée et aboutir à un phénomène autoinflammatoire [4] (table 1).

\section{Epidémiologie}

\subsection{Haplo insuffisance du gène TNFAIP3 (codant la protéine A20)}


L'incidence et la prévalence de cette nouvelle entité autoinflammatoire ne sont pas connues ; à ce jour 51 observations ont été rapportées de façon cosmopolite; la plupart de ces observations sont dites «Behçet-like» du fait de l'aphtose bipolaire [6,8,10-13,20]. Les patients sont majoritairement de sexe féminin (68\%) d'origine japonaise $(60 \%)$, caucasienne (30\%) et turque (10\%) (table 2). Les symptômes commencent dans l'enfance chez la grande majorité des patients $(92 \%)$. Parmi les vingt-deux patients appartenant à 9 familles décrites par Kadowaki et al., 9/22 présentaient un tableau Behçet-like et 13/22 des tableaux plus complexes associant des manifestations autoinflammatoires et une autoimmunité permettant de penser que le nombre de cas non encore identifiés pourrait être assez important [12].

\subsection{Otulinopénie}

Pour l'instant 6 patients seulement ont été décrits, issus de 3 familles consanguines, 2 d'origine turque et une d'origine pakistanaise ; dans une des familles, deux patients sont décédés précocement, probablement du même déficit; les symptômes ont commencé en période néonatale dans les 3 premiers mois de vie, et un patient avait un tableau ressemblant au PRAAS du fait de la présence de lipodystrophie et de panniculite; trois des 6 enfants étaient nés prématurés [7,9].

\section{Spectre clinique (table 1$)$}

\subsection{HA20 (tables 1, 2, 3 et figure 2)}

La description initiale de Zhou et al, est plus détaillée dans une publication récente de la même équipe [6,8]. Les 16 patients sont issus de 7 familles dont 5/7 multiplexes avec une transmission dominante. Chez ces patients la maladie commençait tôt dans la vie avec certaines ressemblances avec la MB du fait de la présence d'aphtose bipolaire chez la majorité des patients ainsi que d'une inflammation oculaire [6] (figure 2). Elle s'en distinguait par la présence de poussées fébriles dans la majorité des cas associées à une inflammation biologique chez tous les patients lors des poussées (non habituelle dans la $\mathrm{MB}$ où l'inflammation systémique n'accompagne que les poussées de sérite, de vascularite et lors des atteintes digestives qui sont par ailleurs rares dans les populations non asiatiques), la prédominance d'uvéites antérieures et la relative fréquence de colite ulcéreuse associée (6/11 
patients) avec diarrhée sanglante. Une autre différence importante est que certains des patients souffraient d'infections ORL et pulmonaires à répétition. Deux patients ont développé une vascularite cérébrale et un patient a présenté une thrombose pulmonaire liée à une vascularite. Certains patients avaient des stigmates d'auto-immunité biologique. Le diagnostic initial était majoritairement une MB mais pour d'autres il s'agissait d'un PFAPA (Periodic Fever Aphtous stomatitis Pharyngitis Adenitis), d'une arthrite juvénile, d'un lupus ou d'une maladie de Crohn [6]. Vingt-deux autres patients avec un HA20 et appartenant à 9 familles sont décrits par Kadowaki et al.; neuf patients d'entre eux présentaient un tableau Behçet-like avec des âges de début dans l'enfance ou l'adolescence, 4/22 avaient débuté comme des PFAPA, 2/22 sous forme d'AJI (arthrite juvénile idiopathique) polyarticulaire; les autres patients présentaient au début une maladie de Kawasaki, une FMF (fièvre méditerranéenne familiale)-like et des aphtoses buccales à répétition[12]. L'association autoimmunité et autoinflammation était présente chez 9/22 patients (en dehors des 9 ayant le tableau Behçetlike) et comportait du psoriasis, des thyroidites autoimmunes, des maladies de Crohn, et un lupus ayant évolué vers un ALPS (syndrome lymphoprolifératif avec autoimmunité). En plus de ces deux séries importantes, 10 autres patients sont décrits avec des tableaux d'aphtose et de fièvre récurrente ce qui porte à 51 le nombre de patients publiés au total à ce jour et résumés dans les tables 2 et 3 . Par souci de clarté, une observation bien différente associant un syndrome polymalformatif, une vascularite récurrente à IgA et une autoimmunité mais sans fièvre récurrente due à une large délétion comprenant 60 gènes sur le chromosome $6 \mathrm{p}$ dont CITED2, IFNGR1 et TNFAIP3 n'a pas été incluse dans la table 3 [21] .

\subsection{Otulinopénie (Otulin-related autoinflammatory syndrome :}

\section{ORAS)}

Le tableau clinique comporte des manifestations inflammatoires systémiques sévères néonatales faites de fièvres prolongées, de gonflements articulaires, d'une diarrhée sévère avec retard de croissance staturopondérale. Les poussées sont longues et ne cèdent pas sans traitement[7,9]. Sur le plan cutané, diverses éruptions sont décrites : érythèmes, pustules, nodules sous cutanés, panniculites et lipodystrophies. La table 1 et la figure 3 résument les atteintes principales des ubiquitinopathies de la voie NFKB par mutation de TNFAIP3 et d'OTULIN. 


\section{Aspects génétiques}

\subsection{HA20}

Le gène TNFAIP3 (tumor necrosis factor $\alpha$-induced protein 3, A20) qui code la protéine A20 se situe sur le chromosome 6 et comporte 9 exons; le premier exon est non codant ; cinq exons contribuent à la formation du domaine OTU (ovarian tumor), 3 exons au domaine doigts de zinc, le dernier exon étant en partie non codant; la protéine correspondante comporte deux domaines principaux un domaine OTU (ovarian tumor) en région $\mathrm{N}$ terminale qui est essentiel à l'activité déubiquitinase de A20 (c'est une cystéine C103 qui confère une K63 deubiquitination.) et un domaine $\mathrm{C}$ terminal en 7 doigts de zinc (7 ZF) qui lui confère une activité d'ubiquitinase $[3,4,8,17,18]$. Les mutations décrites sont pour la plupart des mutations non-sens ou des petites délétions décalant le cadre de lecture, résultant en une troncation du domaine OTU ou dans le domaine ZF altérant la fonction de la protéine A20 ; il n'est pas possible aujourd'hui d'effectuer des corrélations entre le génotype et le phénotype et l'extrême variabilité du tableau clinique et de l'évolution des patients décrits avec HA20 permet de penser que d'autres facteurs comme des gènes modificateurs ou une régulation épigénétique sont probablement impliqués [4]. Il semblerait néanmoins que les patients ayant des mutations dans le domaine ZF d'ubiquitination aient le même phénotype que ceux ayant des mutations dans le domaine OTU [4].

\subsection{Otulinopénie}

Le gène OTULIN (FAM105B) comporte 7 exons et est situé sur le chromosome 5; l'otulinopénie résulte de mutations récessives (faux sens et petites délétions décalant le cadre de lecture) entrânant une perte de fonction. Damgaard et al ont rapporté 3 patients issus d'une même famille consanguine et porteurs d'une mutation homozygote faux sens dans le gène de l'OTULIN (c.815T>C; p.Leu272Pro ; L22P dans l'exon 6) ; cette mutation déstabilise l'otuline et diminue sa fonction; les cellules des patients exprimaient des taux élevés de Met1-Ub en comparaison des contrôles [7]. En plus de cette mutation, deux autres sont décrites dans le domaine OTU par Zhou et al, une délétion dans l'exon 5 (c.517delC) et une substitution dans l'exon $6(\mathrm{c} .731 \mathrm{~A}>\mathrm{G})[9]$. 


\section{Diagnostics différentiels}

\subsection{HA2O}

\subsubsection{Maladies autoinflammatoires polygéniques}

\subsubsection{1. $\quad$ Maladie de Behçet (MB)}

Le principal diagnostic différentiel est la MB, particulièrement dans sa forme pédiatrique, il existe cependant des différences représentées sur la figure 4. Un début très précoce, une transmission d'allure dominante et la présence de poussées fébriles doivent faire suspecter un HA20. Dans la MB sporadique, le début est plus tardif (vers l'âge de 30 ans), les aphtes buccaux et génitaux évoluent en général sans contexte fébrile et inflammatoire et les aphtes buccaux ne laissent pas de cicatrice. Les uvéites du Behçet sporadique sont plutôt postérieures ou totales et rarement antérieures isolées et la vascularite rétinienne prend un aspect hémorragique formant des feuilles de fougère. Les signes digestifs de la MB sont généralement discrets (douleurs abdominales isolées) et il existe rarement une colite hémorragique en rapport avec des ulcérations aphtoïdes au premier plan. L'attente articulaire de HA20 (polyarthrite) est très différente des atteintes du Behçet qui sont plutôt des oligoarthrites touchant les membres inférieurs [6].

\subsubsection{Syndrome PFAPA}

Un syndrome de fièvre périodique avec adénites cervicales, pharyngite exsudative et aphtes peut éventuellement masquer un HA20. Dans sa forme habituelle pédiatrique (enfants de moins de cinq ans), l'aphtose buccale reste mineure et il n'y a pas d'aphtose génitale. Les signes digestifs sont réduits à des douleurs abdominales liées à des ganglions mésentériques dans le PFAPA ; on n'y observe ni diarrhée, ni colite. L'évolution du PFAPA est en principe transitoire (quelques mois à années) avec la réserve que ce syndrome est parfois un mode d'entrée dans une pathologie inflammatoire plus complexe : authentique MB voir HA20 [22].

\subsubsection{AJI systémique}

La maladie de Still de l'enfant est une maladie autoinflammatoire marquée par des poussées fébriles de durée longue, associée à des éruptions maculeuses et à des atteintes articulaires allant d'arthralgies/myalgies simples à une polyarthrite destructrice. Elle n'a pas de marqueur de diagnostic de certitude fiable. Comme pour le syndrome PFAPA, certains patients 
rapportés par Kadowaki sont entrés dans leur maladie par des manifestations «Still-like » et ont évolué au cours du temps vers un profil plus autoimmun de type rhumatisme psoriasique [23].

\subsubsection{Les maladies inflammatoires monogéniques du côlon et de l'intestin}

À côté des formes familiales de la maladie de Crohn correspondant à des mutations du gène NOD2/CARD15 et dont la reconnaissance est favorisée par l'histologie, un certain nombre de colites ulcéreuses sont liées à des affections monogéniques. Celles-ci débutent en période néonatale ou dans les premiers mois de vie avec une sévérité pouvant parfois nécessiter une greffe de moelle allogénique. Parmi celles-ci il faut évoquer en fonction des signes associés des déficits immunitaires primitifs comme l'IPEX (X-Linked immuno-dysregulation, polyendocrinopathy, enteropathy, ) et le XIAP (X-linked inhibitor apoptotosis) I, ou des causes autoinflammatoires comme les déficits en IL10 et IL10 R et le déficit en mévalonate kinase.

\subsubsection{Les autres maladies autoinflammatoires monogéniques}

Les fièvres récurrentes historiques comme le déficit en mévalonate kinase, les cryopyrinopathies, et la FMF peuvent donner des aphtes; le syndrome TRAPS (TNF Receptor Associated Periodic Syndrome) peut entrainer des poussées de fièvre longues [1]. Il faut bien veiller à faire un arbre généalogique et connaitre l'origine géographique des patients.

\subsection{Otulinopénie}

\subsection{Maladies autoinflammatoires polygéniques}

Certaines maladies inflammatoires chroniques intestinales à début précoce peuvent ressembler à une otulinopénie [1]. Des dermatoses neutrophiliques et le syndrome de Sweet sont également un diagnostic différentiel avec les signes cutanés[24]

\subsection{Maladies autoinflammatoires monogéniques}

Le syndrome de Majeed par mutation de LPIN2 donne une dermatose neutrophilique à début néonatal [1]. Les maladies de Crohn monogénique peuvent mimer une otulinopénie. Enfin, le syndrome autoinflammatoire avec dermatose neutrophilique associé à la pyrine (PAAND), 
peut donner une atteinte cutanée, digestive voir cardiaque similaire [25]. Le syndrome PRAAS est lié à des mutations ponctuelles (homozygotes, hétérozygotes composites, doubles hétérozygotes, ou hétérozygotes simples) entrainant une perte de fonction du protéasome constitutif (PMSB4, PMSA3), du protéasome inductible ou immunoprotéasome (PSMB8, PSMB9) ou de la protéine chaperonne (POMP). Toutes les mutations décrites altèrent l'assemblage des différentes sous unités et donc la fonction essentielle qui est de dégrader les protéines $[1,2,26]$. Les patients présentent une fièvre hectique débutant en période néonatale, des éruptions de type panniculite avec lipoatrophie progressive, de la myosite, des éruptions périorbitaires violacées et des calcifications cérébrales et vasculaires. Une CRP élevée, une hypergammaglobulinémie et une autoimmunité biologique sont fréquentes. Toutefois, l'atteinte digestive sévère de l'otulinopénie n'y est pas présente.

\section{Aspects thérapeutiques}

Les données sur les traitements appliqués et leur efficacité restent assez limitées et sont extraites d'expériences de cas rétrospectifs.

\subsection{HA20}

Tous les patients décrits ont nécessité des traitements allant de la colchicine seule (efficace chez 8 patients), jusqu'à des associations de corticoïdes et d'immunosuppresseurs (méthotrexate, azathioprine, thalidomide, ciclophosphamide, tofacitinib). Globalement les corticoïdes étaient efficaces mais avec des niveaux de corticodépendance élevés occasionnant des effets secondaires majeurs. Les biothérapies utilisées étaient l'anakinra, le tocilizumab, et les anti-TNF et étaient considérées comme pouvant avoir un effet sur l'inflammation systémique. Des traitements substitutifs par IgIV ont été utilisés chez les quelques patients avec déficit immunitaire humoral associé $[6,8,10-13,20]$. Il est probable que pour les patients avec HA20 une approche thérapeutique personnalisée basée en partie sur les taux de cytokines détectées, soit la plus adaptée car il existe probablement une grande variabilité phénotypique qui complexifie l'établissement d'une prise en charge standardisée. Un patient présentant des manifestations sévères à type de vascularite cérébrale a eu une greffe de moelle allogénique puis a récidivé en postgreffe sur un mode lupus-like; un autre patient est décédé de complications digestives hémorragiques $[6,12]$. 


\subsection{Otulinopénie}

Les patients atteints d'ORAS rapportés dans la littérature ont été traités principalement (5/6) par des anti TNF ; un patient sur les six a été traité par des corticoïdes et de l'anakinra [7,9].

\section{Aspects évolutifs}

\subsection{HA20}

Globalement les patients atteints de HA20 évoluent au cours de leur vie en passant d'un profil autoinflammatoire à un profil autoimmun et inversement ; deux patients de la littérature ont évolué vers un syndrome ALPS, syndrome habituellement attribué à des mutations affectant l'apoptose (Fas/FasL)[3,12,19,27]. Le phénotype inflammatoire semble au-devant de la scène et les patients sont généralement réfractaires aux traitements. La variabilité du phénotype au cours de la vie complexifie un peu plus la prise en charge qui semble s'approcher de la médecine personnalisée $[4,6,12]$.

\subsection{Otulinopénie}

L'évolution à long terme n'est pas connue mais il semblerait que les patients sous traitement par anti TNF ou anti IL-1 aient récupéré une croissance normale et soient correctement contrôlés sur le plan inflammatoire ; cette affection ne comportant pas de déficit immunitaire, les infections constatées ont été attribuées aux traitements immunosuppresseurs $[4,7,9]$.

\section{Conclusion}

Cette revue décrivant une nouvelle voie possible pour l'autoinflammation nous rappelle une fois de plus les limites de la médecine purement clinique en revisitant bien des entités comme le syndrome/MB, le syndrome PFAPA et l'arthrite juvénile. Elle nous remet en perspective l'importante intrication de l'autoinflammation avec l'autoimmunité, (c'est-à-dire immunités innée et adaptative) un même défaut apparaissant pouvoir donner chez un même individu au cours de sa vie un PFAPA puis une MB voire un syndrome ALPS. L'extrême hétérogénéité des patients portant parfois une même mutation ouvre aussi la voie à une médecine 
personnalisée pour mieux comprendre les mécanismes physiopathologiques faisant intervenir probablement d'autres gènes modificateurs et pour mieux cibler les traitements.

\section{Conflits d'intérêts}

IKP, SGL, VH ont reçu dans le passé des émoluments pour des expertises ou des conférences pour les laboratoires Novartis et Sobi sans lien avec cette revue. 


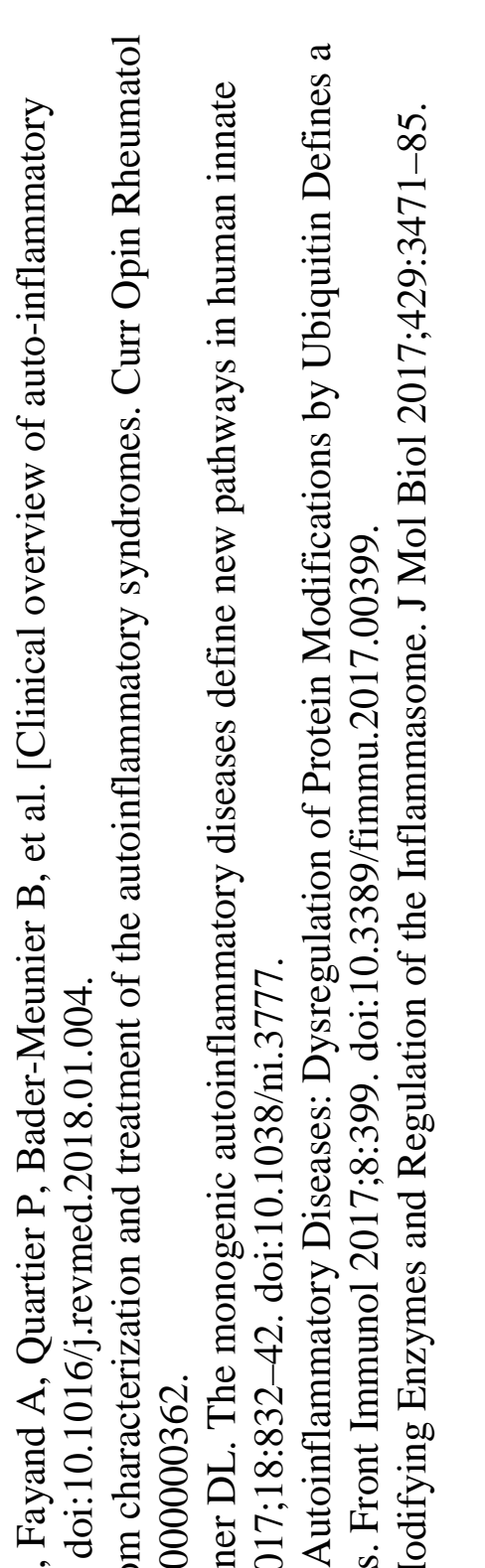

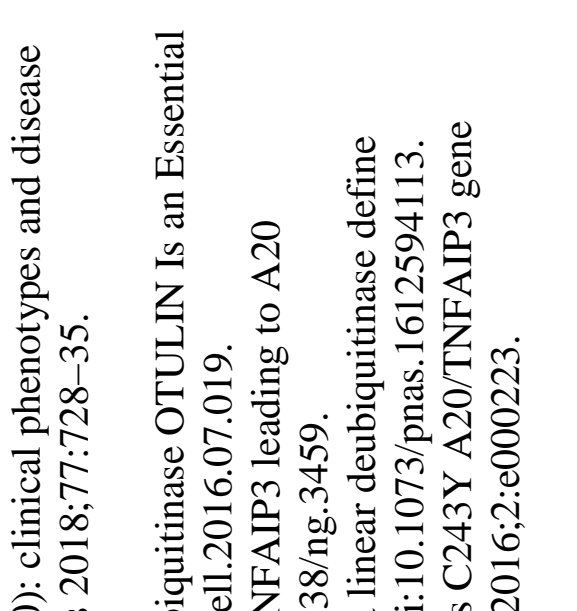

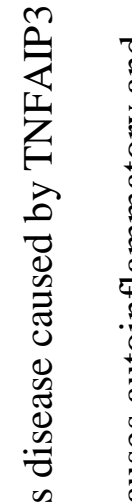

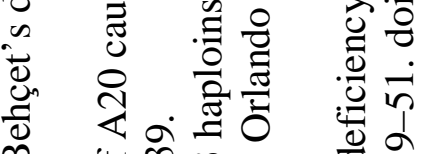

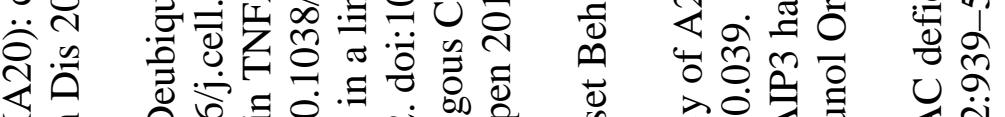

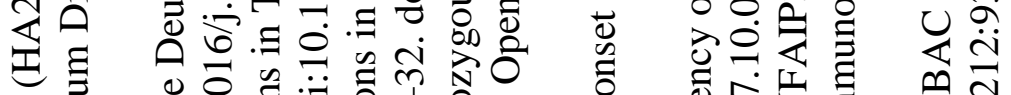

त̄

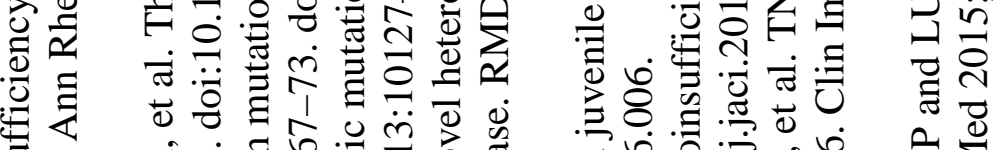

苛

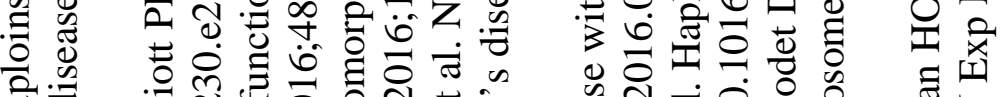

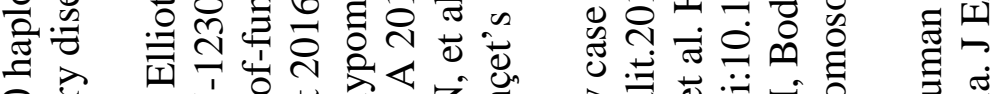

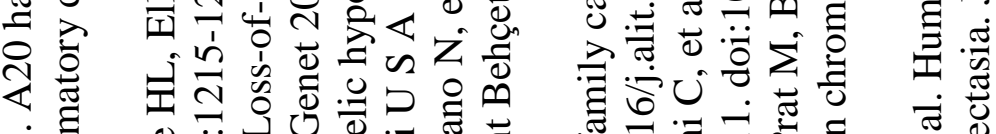

宁言

ค.

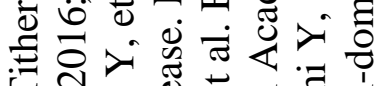

๘ँच

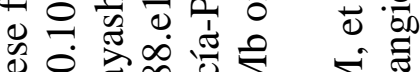

言

荧

$\forall$, no

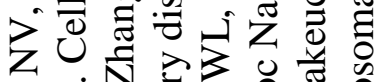

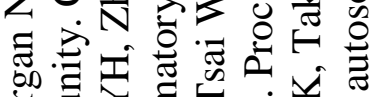

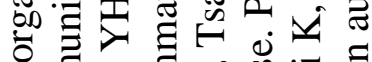

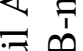

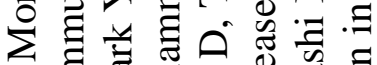

踏

$\sum \frac{\pi}{2}$

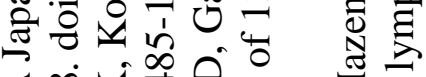

《oi

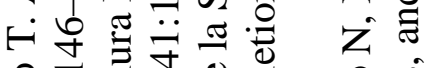

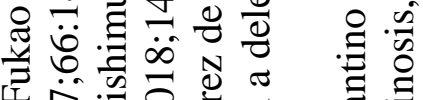

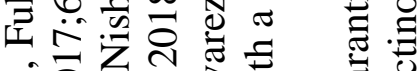

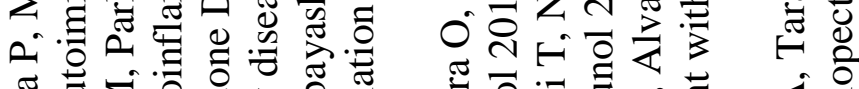

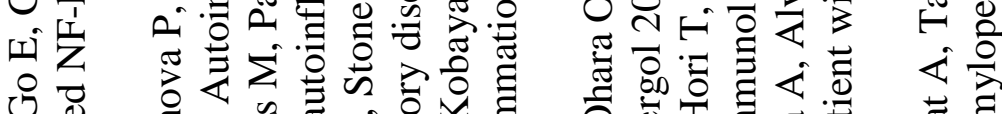

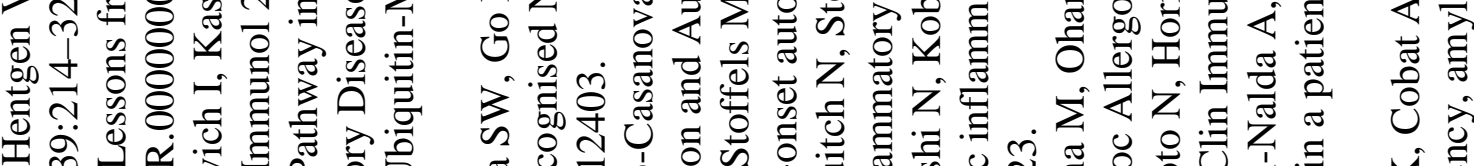

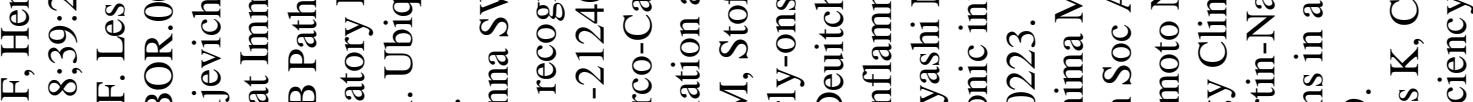

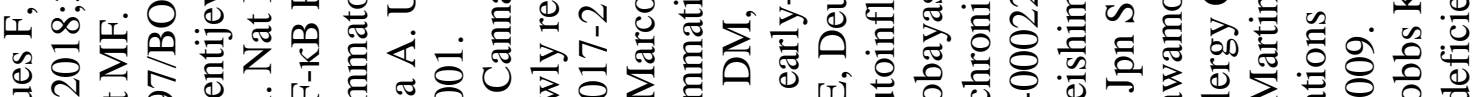

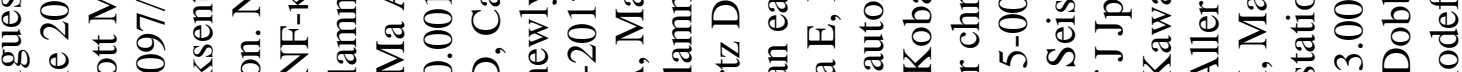
of o 응

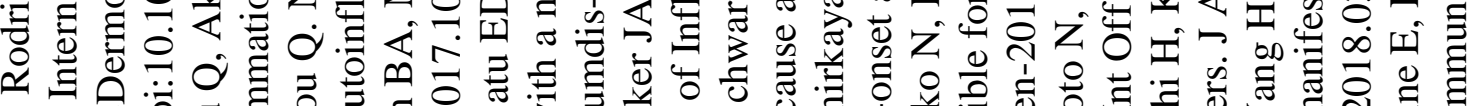

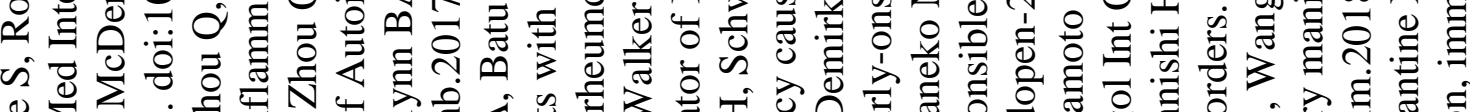

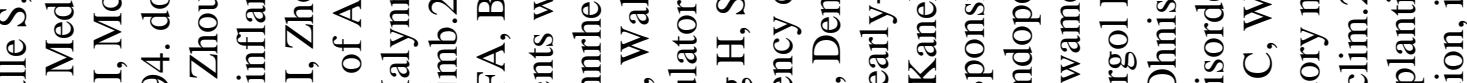

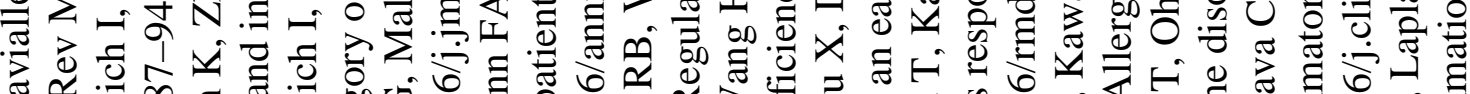

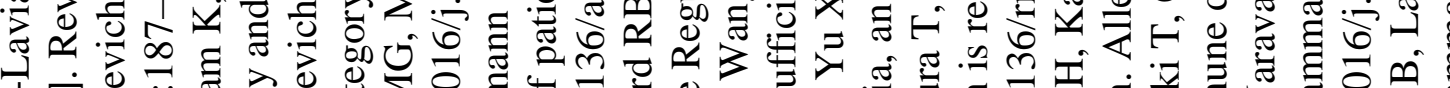

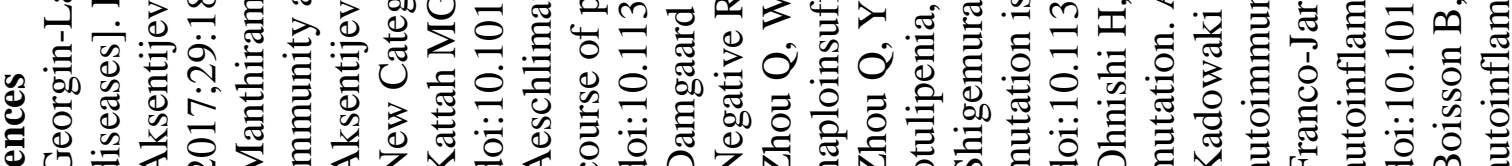

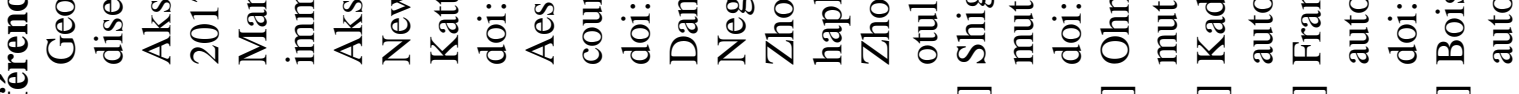

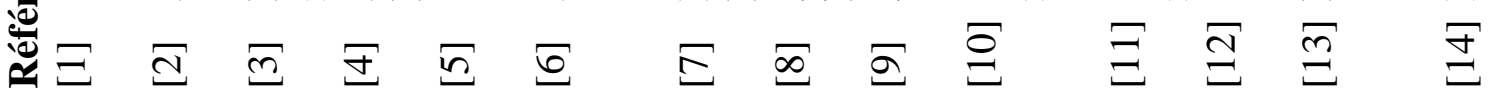




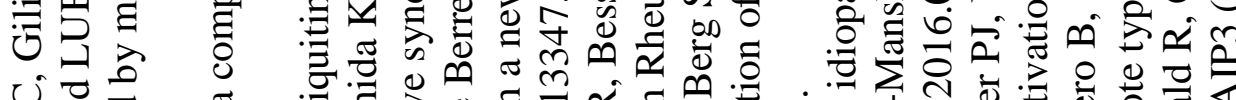

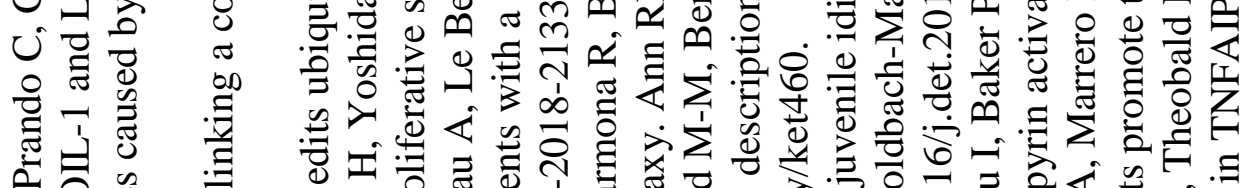

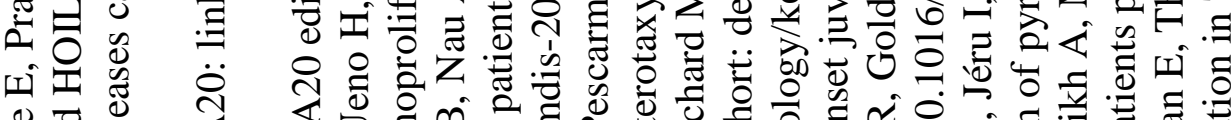

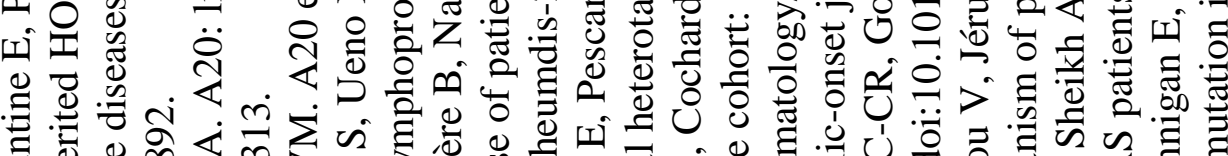

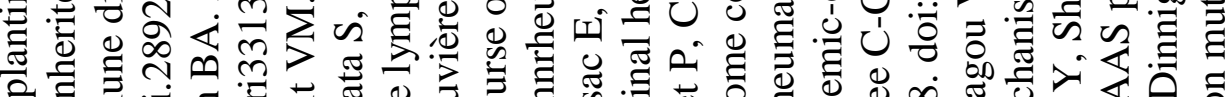

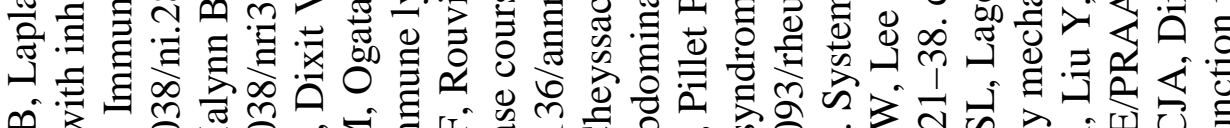
ص

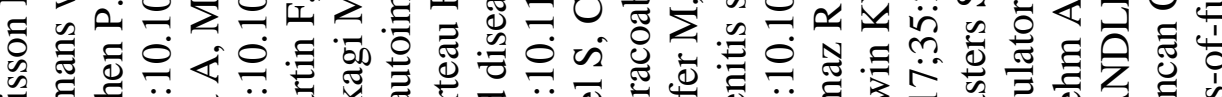

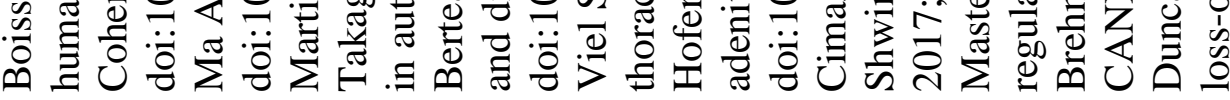

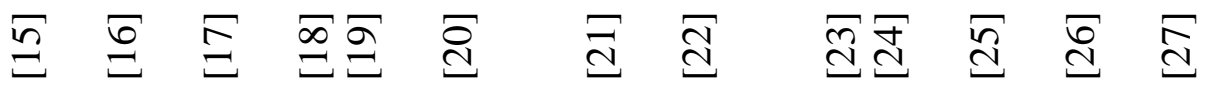




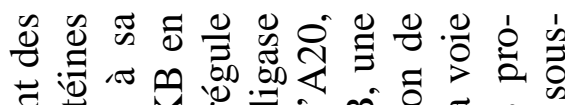

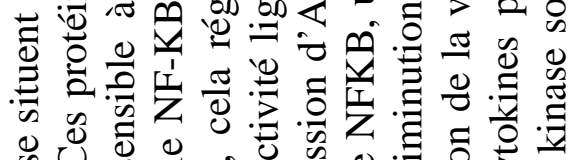

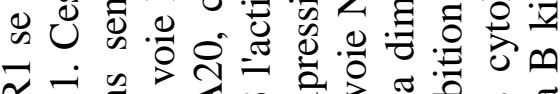

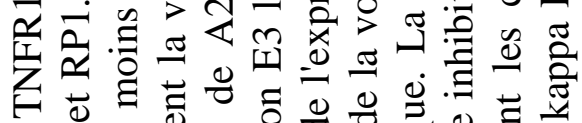

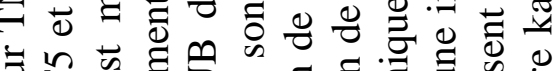

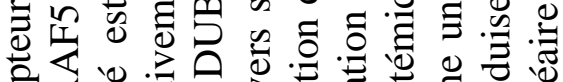

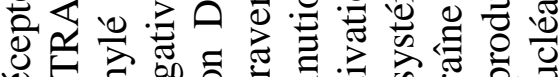

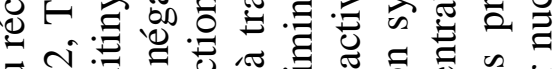

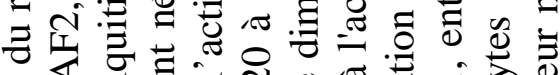

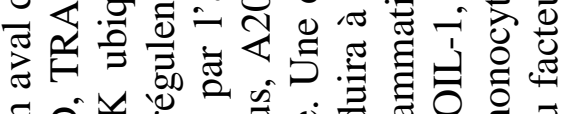

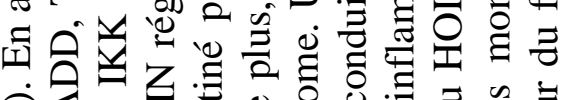

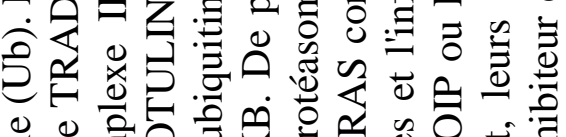

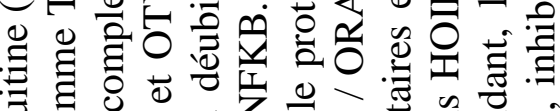

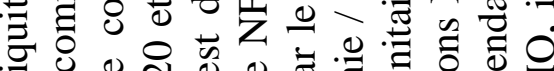

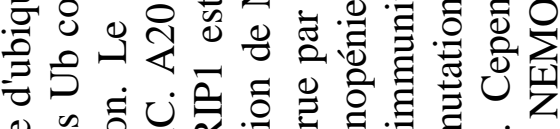

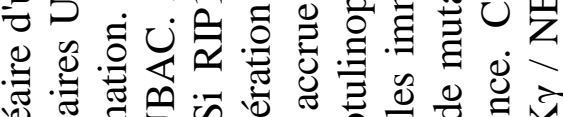

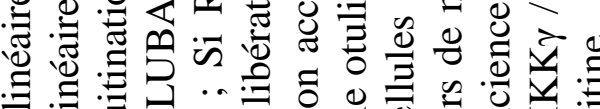

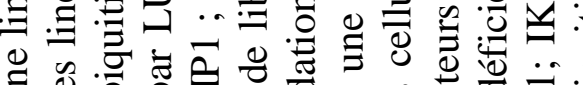

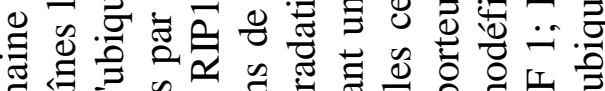

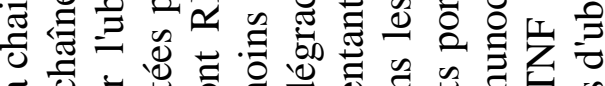

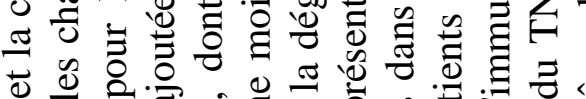

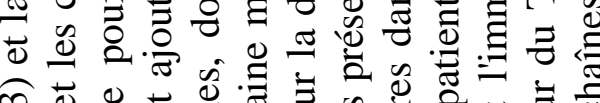

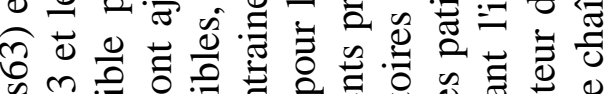

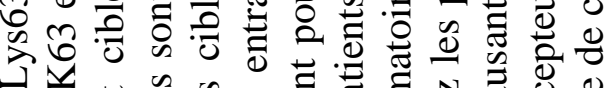

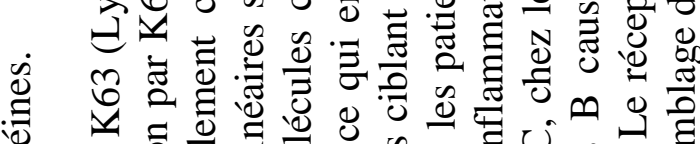

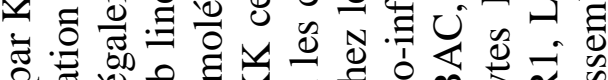

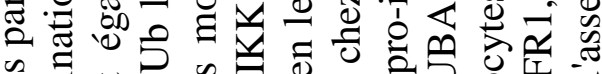

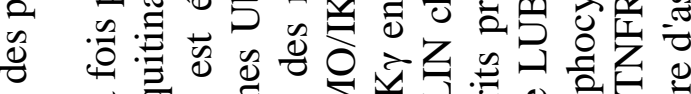

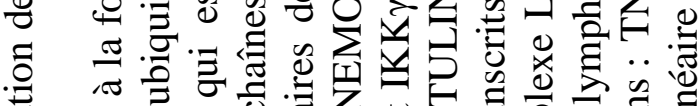

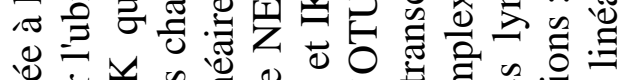

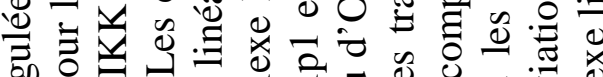
언

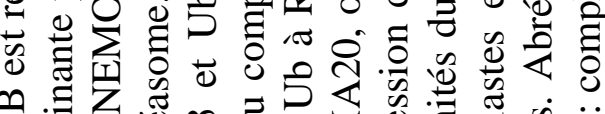

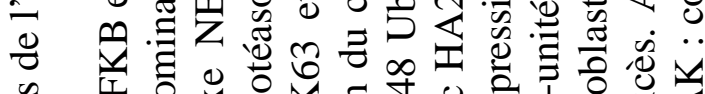
嵌

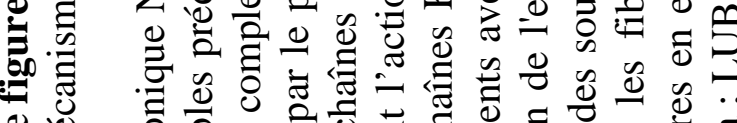

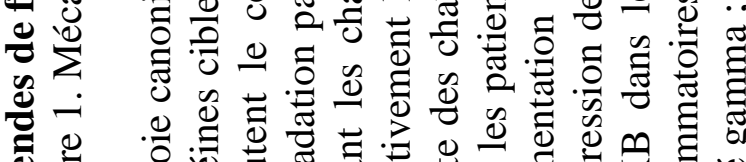

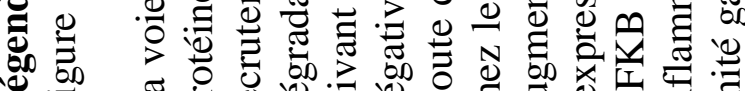

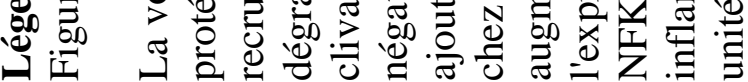




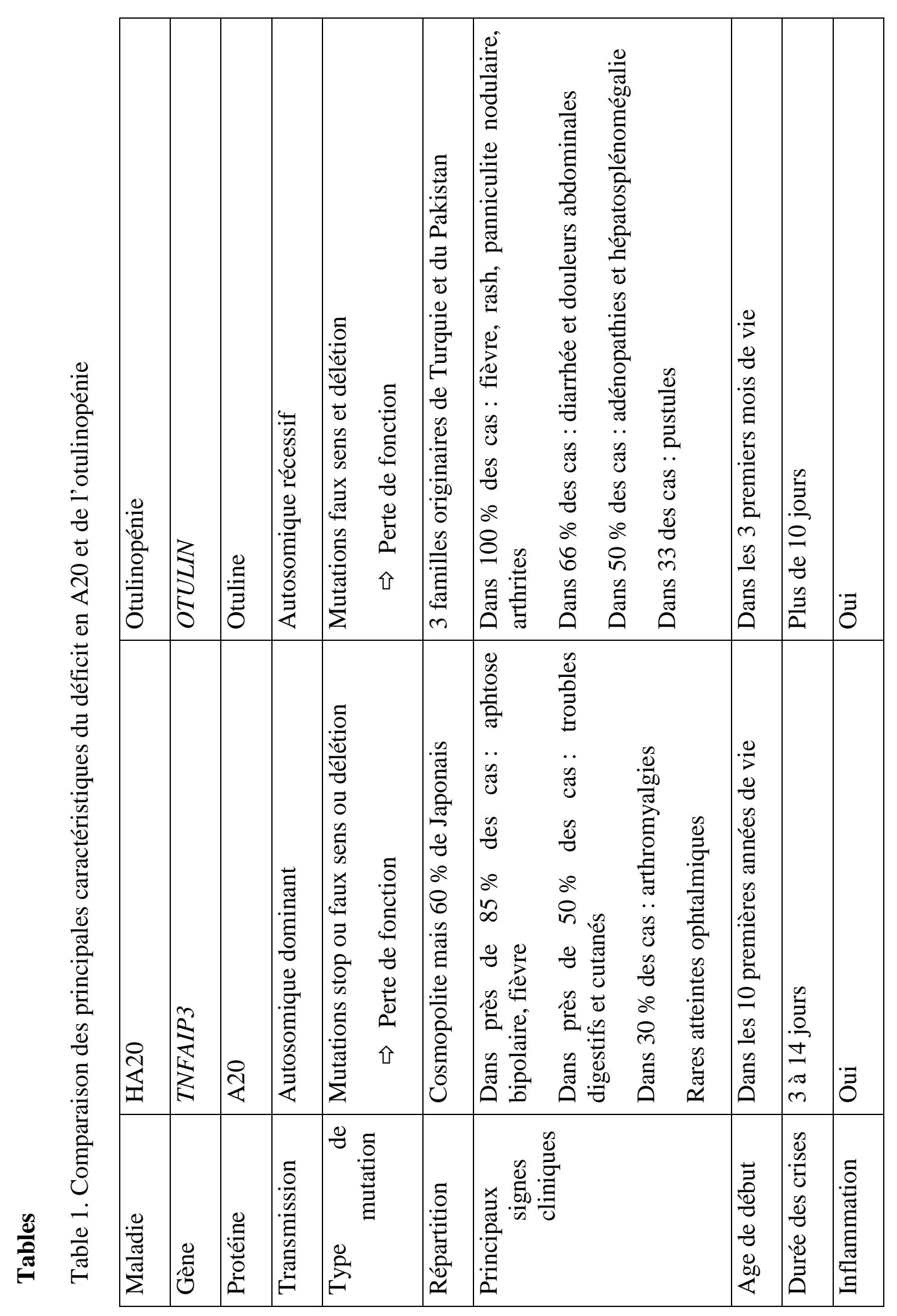




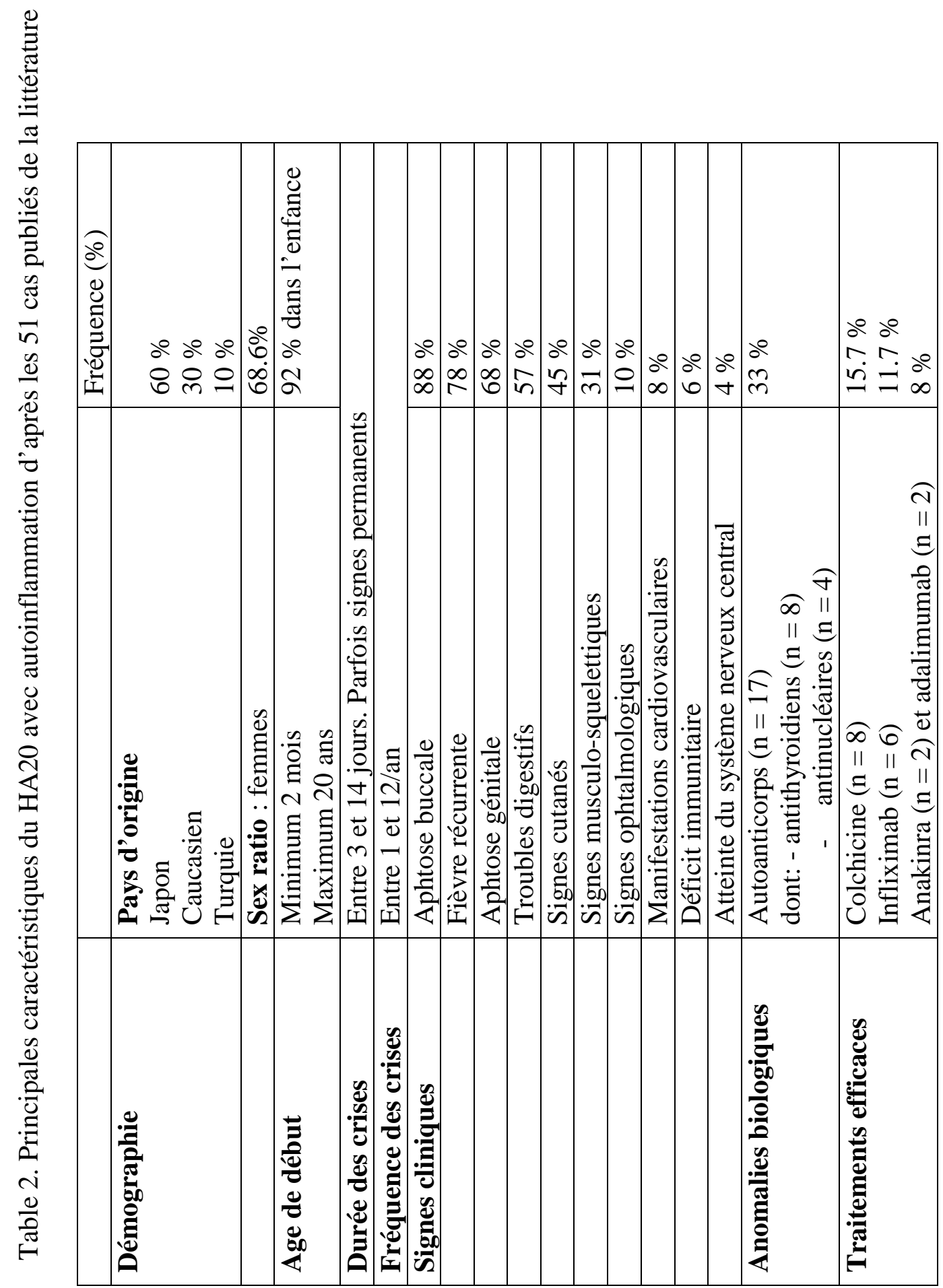




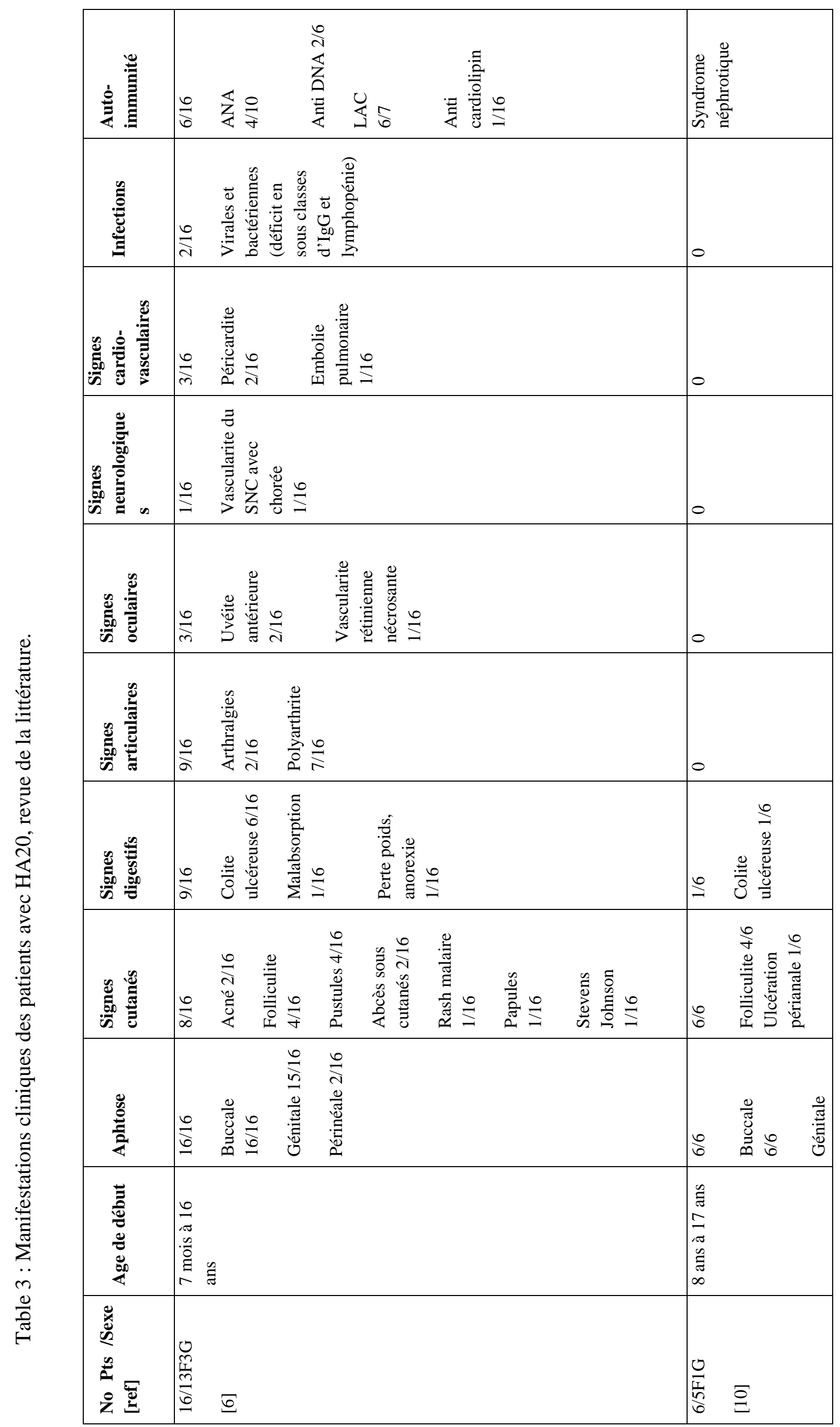




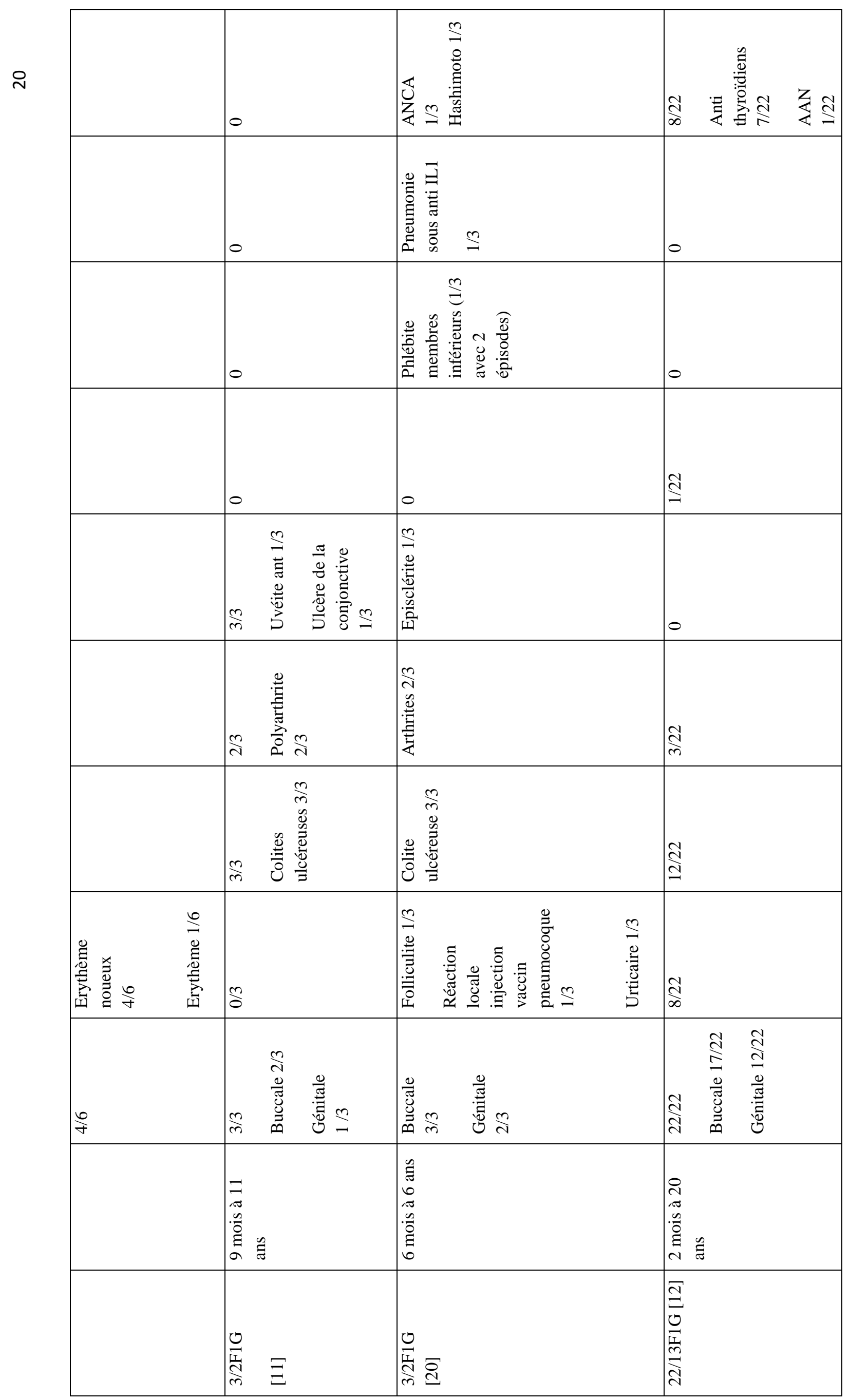




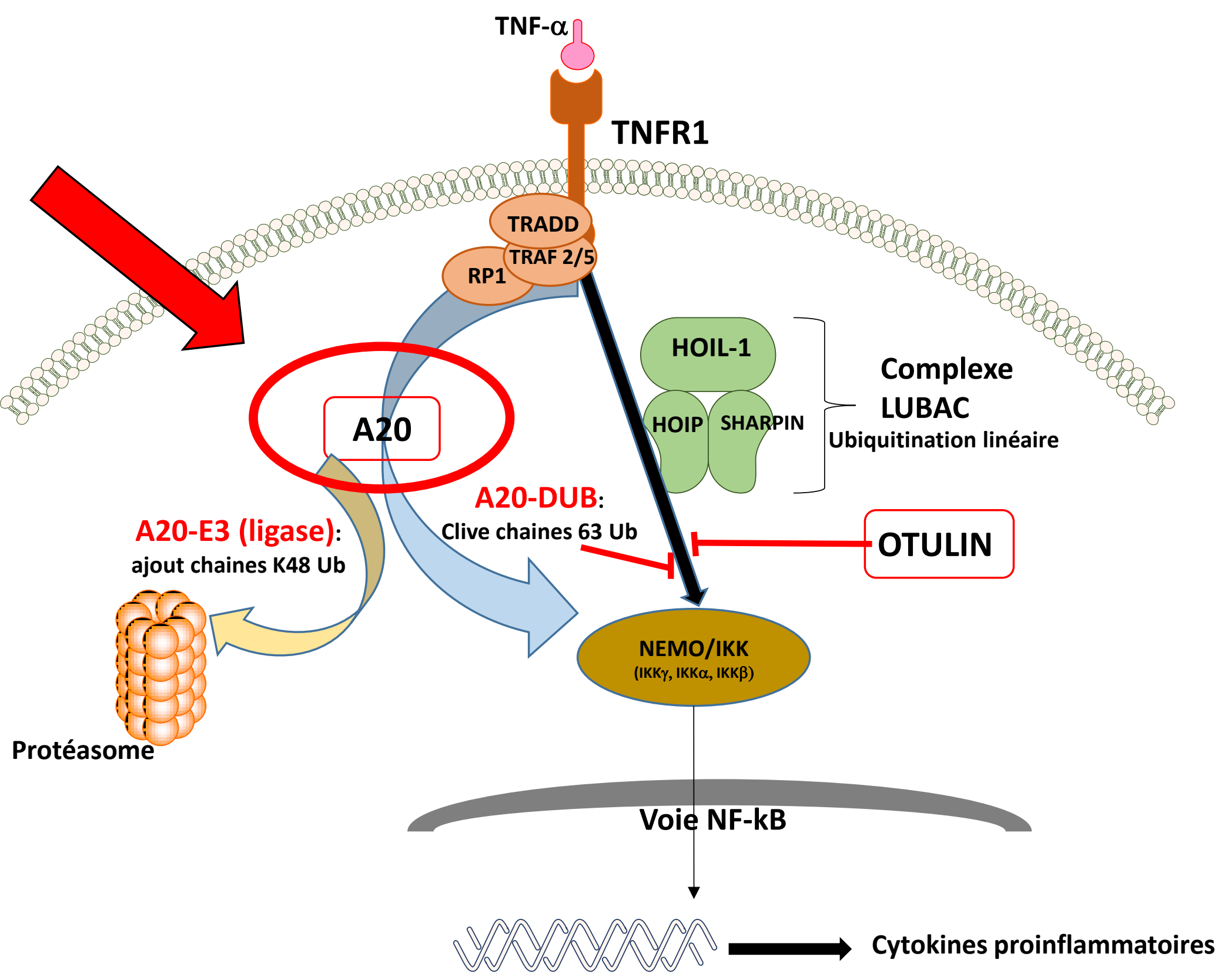




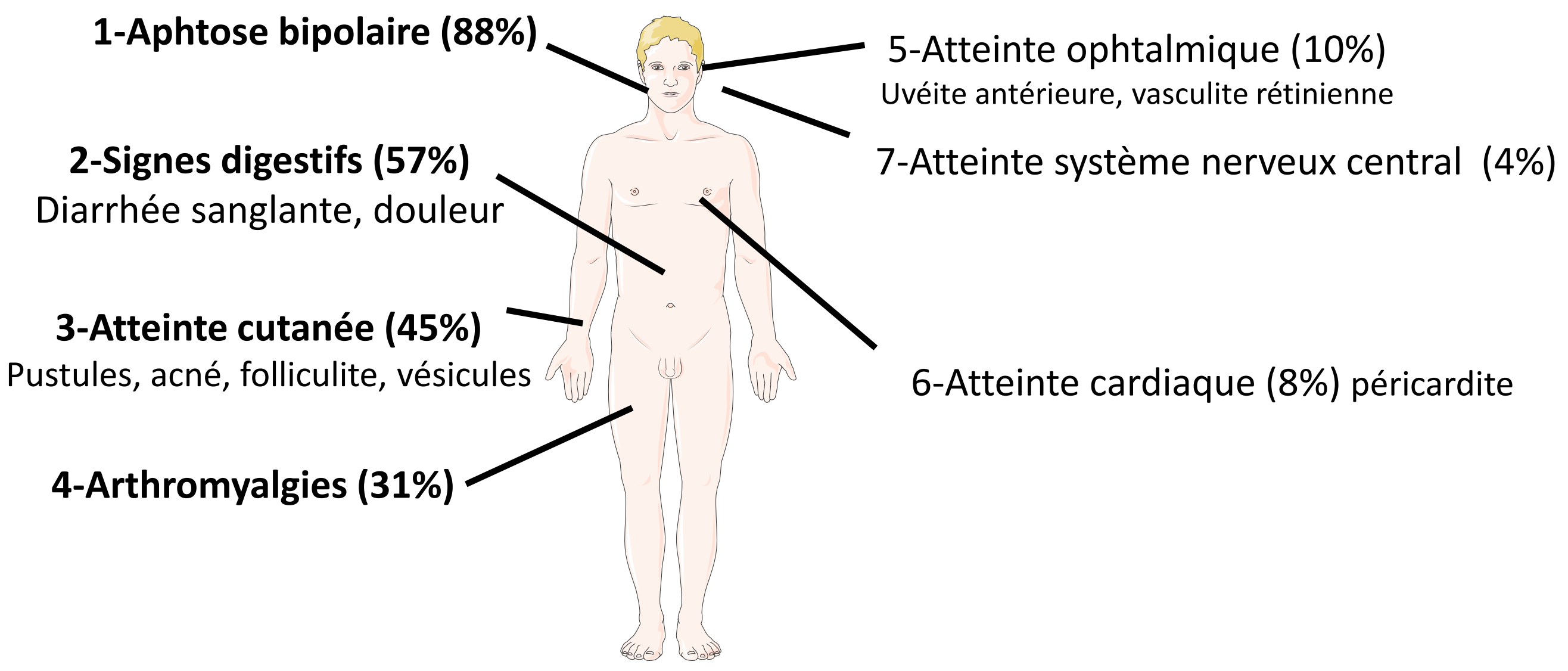




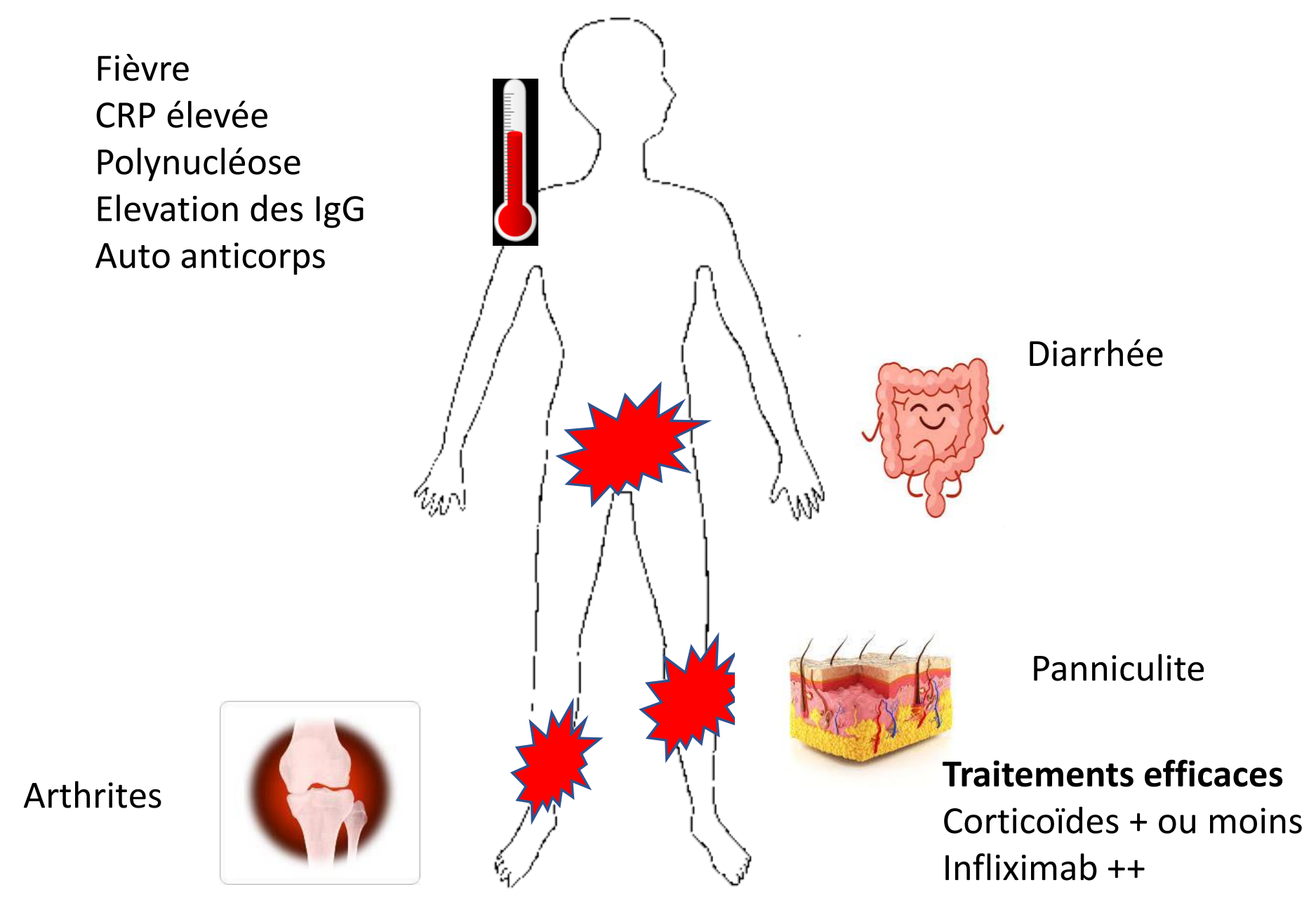




\section{HA20}

Début presque toujours pédiatrique autosomique dominant

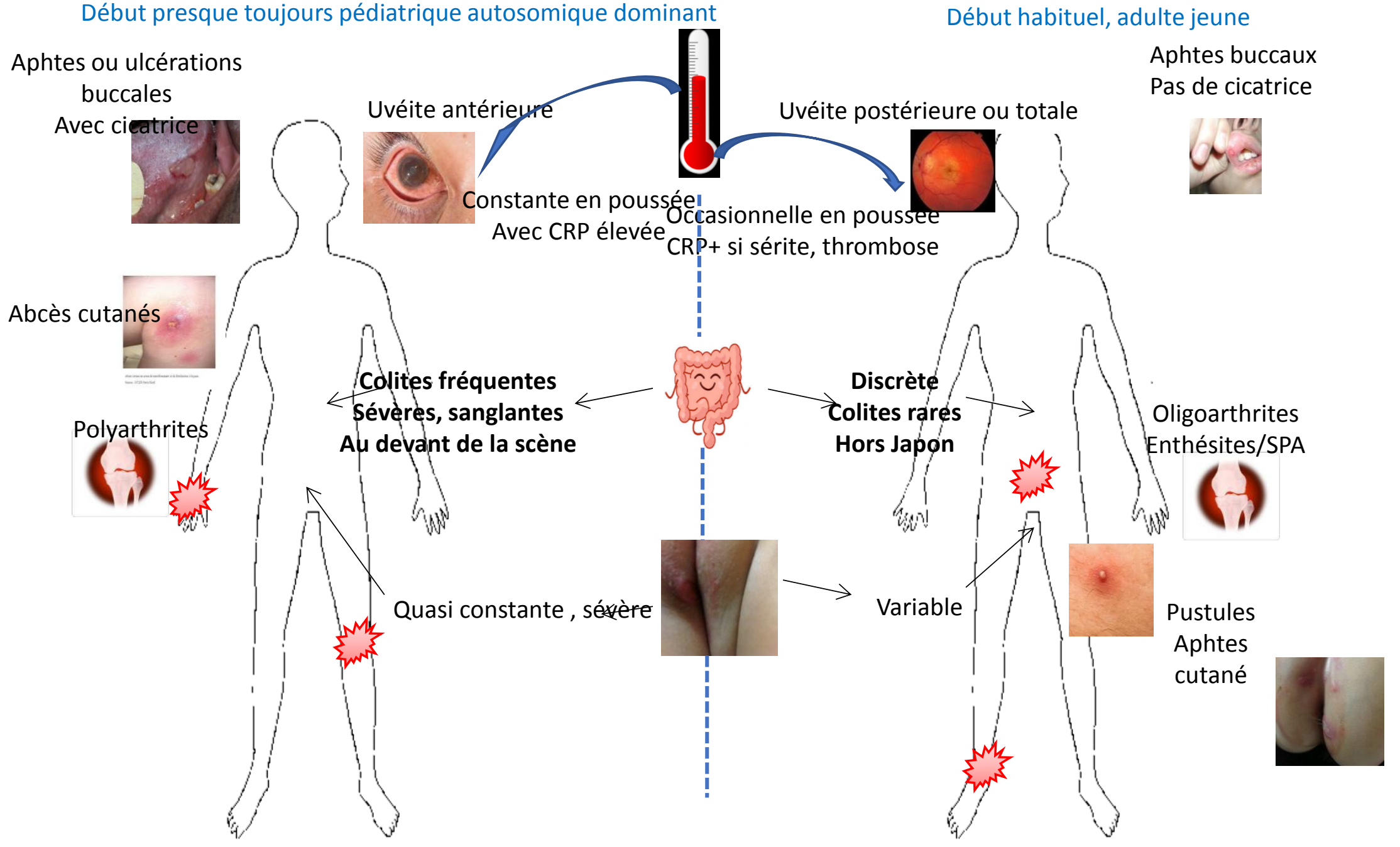

